\title{
Glioblastoma Stem Cells and Comparison of Isolation Methods
}

\author{
Tolga Turan Dundar ${ }^{\mathrm{a}, \mathrm{e}}$, Mustafa Aziz Hatiboglu ${ }^{\mathrm{a}}$, Zehragul Ergulb, c, \\ Mehmet Hakan Seyithanoglua, Elif Sozen ${ }^{b, c}$, Saffet Tuzgen ${ }^{d}$, \\ Mehmet Yasar Kaynard, Erdal Karaoz ${ }^{\text {b, c }}$
}

\begin{abstract}
Background: Glioblastoma (GBM) is the most aggressive and the most common primary brain tumor. Over the last few years, studies have identified many genetical and phenotypical molecular situations for developing new treatment modalities in patients with GBM. Nevertheless, main problem for the GBM is radio-chemotherapy resistance and relapse after the surgery. The identification of glioma stem cells and microenvironmental influences has created a paradigm shift in targets of therapy. Current studies have shown that glioma stem cell is responsible for aggressiveness, recurrence and resistance to therapy of GBM. GBM stem cell isolated from human GBM multiforme fresh tissue samples is important both for curative therapeutic options and personalized targeted therapy. The purpose of this study was to determine the most suitable isolation method of GBM stem cells (GSCs).
\end{abstract}

Methods: Tumor tissue sample was obtained during the surgical resection of lesion in patients with the diagnosis of GBM. Tumor stem cell isolation from tissue was performed in three different ways: 1) GBM cell isolation with trypsin; 2) GBM cell isolation with brain tumor dissociation Kit (BTD Kit); and 3) GBM cell isolation with tumor dissociation enzyme (TDE).

Results: We showed that GSCs were isolated from tumor specimen using flow cytometry and immunofluorescence staining. Our study showed that isolation with BTD Kit is the most suitable method to isolate GBM tissue-derived glial tumor stem cells.

Manuscript submitted February 11, 2019, accepted March 16, 2019

aDepartment of Neurosurgery, Faculty of Medicine, Bezmialem Vakif University, Istanbul, Turkey

${ }^{\mathrm{b}}$ Center for Regenerative Medicine and Stem Cell Research and Manufacturing, Liv Hospital, Istanbul, Turkey

'Department of Histology and Embryology, Faculty of Medicine, Istinye University, Istanbul, Turkey

dDepartment of Neurosurgery, Cerrahpasa Medical Faculty, Istanbul University, Istanbul, Turkey

${ }^{\mathrm{e}}$ Corresponding Author: Tolga Turan Dundar, Department of Neurosurgery, Faculty of Medicine, Bezmialem Vakif University, Adnan Menderes Bulvari, Vatan Caddesi, Fatih, Istanbul 34093, Turkey.

Email: tdundar@bezmialem.edu.tr

doi: https://doi.org/10.14740/jocmr3781
Conclusions: The development of alternative personalized therapies targeting brain tumor stem cell is urgently needed. It is important to understand the fundamental mechanisms of driving stem cells. If their life cycle mechanisms can be identified, we can control the growth of GBM.

Keywords: Glioblastoma; Cancer stem cell; Targeted therapy

\section{Introduction}

Gliomas are the brain tumors that resemble normal stromal (glial) cells of the brain, such as astrocytes (astrocytomas), oligodendrocytes (oligodendrogliomas) and ependymal cells (ependymomas). Among gliomas, glioblastoma (GBM; WHO grade IV astrocytoma) is the most aggressive and the most common primary brain tumor. Moreover, GBM is heterogeneous and may have significant vascularization $[1,2]$. Recently, GBMs were described as isocitrate dehydrogenase (IDH)wildtype, IDH-mutant type and nitric oxide synthase (NOS)positive type [2].

There is another classification system for GBM. This classification includes subtypes based on clinical and molecular characteristics. These subtypes are classical, mesenchymal, proneural, and neural. Proneural subgroup that includes the amplification of CD133 marker does not respond to treatment [3].

Current treatment regimens are maximal safe surgical resection, radiotherapy, and chemotherapy [4]. Over the last few decades, various therapies have been studied and tested clinically. Despite extensive molecular and genetic analyses of GBM, the median survival is only about $12-14$ months $[5,6]$. New treatment regimens, including targeting cells responsible for tumor growth or progression and signaling pathways, are required for more effective treatment in patients with GBM [1].

GBM stem cell (GSC) has been found to play a crucial role in development and growth of GBM. Several studies have shown that GSC is responsible for cancer aggressiveness, tumor recurrence and tumor resistance to conventional therapies including radiation therapy and chemotherapy $[7,8]$. Recent studies have focused on isolating and understanding the biology of GSC and finally targeting GSC in order to provide effective treatment for patients with GBM $[4,9,10]$. 
Incorporation of GSC's signaling pathways and development of new molecular therapies will further advance tailoring personalized therapy for GBM. Therefore, isolating GSC from fresh tumor specimen, which is a difficult procedure, is crucial to conducting further studies in this area. This study aims to determine the most appropriate isolation method for obtaining GSC from fresh tumor specimen.

\section{Materials and Methods}

This study was approved by IRB (No: B.30.2.BAV.0.05/183). All procedures performed in studies involving human participants were in accordance with the ethical standards of the institutional and/or national research committee (Bezmialem Foundation University/Human Ethical Committee) and with the 1964 Helsinki declaration and its later amendments or comparable ethical standards.

Fresh tumor specimens were obtained from patients who underwent surgical resection of histologically confirmed GBM.

\section{Isolation and culture of tumor stem cells}

Tumor specimens were immediately delivered to laboratory in phosphate buffered saline (PBS) solution containing 10-15\% penicillin/streptomycin. GSC isolation from fresh specimen was performed in three different ways: 1) GBM cell isolation with trypsin; 2) GBM cell isolation with brain tumor dissociation Kit (BTD Kit, Miltenyi Biotec, Bergisch Gladbach, Germany); and 3) GBM cell isolation with tumor dissociation enzyme (TDE, Miltenyi Biotec, Bergisch Gladbach, Germany).

\section{GBM cell isolation with trypsin}

Tumor specimen was dissociated into small pieces and the tissue pieces were placed in trypsin-ethylenediaminetetraacetate (EDTA) solution $(0.25 \%$, Gibco/Life Sciences, Carlsbad, CA, USA) and incubated for $10-15 \mathrm{~min}$ in a $37^{\circ} \mathrm{C}$ water bath. At the end of the incubation period, trypsin activation was stopped by adding to the tissue from medium containing $10 \%$ fetal bovine serum (FBS). Centrifugation was performed to recover the cells from the enzyme. Medium was added to the pellet, and the cells were filtered with a 70-micron cell strainer and washed by centrifugation. A total of $1 \times 10^{6}$ cells were seeded in each cell culture flask with a medium composed of Dulbecco's modified Eagle's Medium (DMEM)/F12 medium (Gibco, Carlsbad, CA, USA), 10\% FBS (Gibco, Carlsbad, CA, USA), $100 \mathrm{U} / \mathrm{mL}$ penicillin, and $0.1 \mathrm{mg} / \mathrm{mL}$ streptomycin (Gibco, Carlsbad, CA, USA) at $5 \% \mathrm{CO}_{2}$ atmosphere and $37{ }^{\circ} \mathrm{C}$. For suspension culture and adherent culture, T25 ultra-low attachment flasks and T25 cell culture flasks were used, respectively.

\section{GBM cell isolation with BTD Kit}

Tumor specimen was dissociated into small pieces, Hank's buffered salt solution (HBSS) was added on the tissue and it was transferred to a centrifuge tube. After centrifugation, the supernatant was discarded and added to the tissue from buffer $\mathrm{X}$ in the BTD Kit (Miltenyi Biotec, Bergisch Gladbach, Germany) [11]. The enzyme N, enzyme A and buffer $\mathrm{Y}$ in the kit were added at specified ratios to the centrifuge tube and mixed. The tissue was incubated in this solution for 15 min with gentle shaking at $37^{\circ} \mathrm{C}$. At the end of the period, the tissue was mechanically dissociated and allowed to incubate for $10 \mathrm{~min}$. After the tissue was dissociated it was filtered with a 70-micron cell strainer and centrifuged. Cells were washed by centrifugation two more times with HBSS and $1 \times 10^{6}$ cells were seeded in each cell culture flask with a medium composed of DMEM/F12 medium (Gibco, Carlsbad, CA, USA), 10\% FBS (Gibco, USA), $100 \mathrm{U} / \mathrm{mL}$ penicillin, and $0.1 \mathrm{mg} / \mathrm{mL}$ streptomycin (Gibco, Carlsbad, CA, USA) at 5\% $\mathrm{CO}_{2}$ atmosphere and $37{ }^{\circ} \mathrm{C}$. For suspension culture and adherent culture, T25 ultra-low attachment flasks and T25 cell culture flasks were used, respectively.

\section{GBM cells isolation with TDE}

Tumor specimen was dissociated into small pieces, the tissues were transferred to a centrifuge tube and TDE (DCS innovative diagnostic system, oncogram kit) was added. The tube was allowed to incubate for $1-2 \mathrm{~h}$ at $37^{\circ} \mathrm{C}$ by shaking gently (until the tissue was completely disintegrated). At the end of the incubation period, the medium was added, filtered with a 70-micron cell strainer and washed twice by centrifugation. A total of $1 \times 10^{6}$ cells were seeded in each cell culture flask with a medium composed of DMEM/F12 medium (Gibco, Carlsbad, CA, USA), 10\% FBS (Gibco, Carlsbad, CA, USA), $100 \mathrm{U} /$ $\mathrm{mL}$ penicillin, and $0.1 \mathrm{mg} / \mathrm{mL}$ streptomycin (Gibco, Carlsbad, CA, USA) at $5 \% \mathrm{CO}_{2}$ atmosphere and $37^{\circ} \mathrm{C}$. For suspension culture and adherent culture, T25 ultra-low attachment flasks and T25 cell culture flasks were used, respectively.

GSCs were visualized by phase-contrast microscopy after generating spheres and adhering in culture dishes on day 4 .

\section{Flow cytometry analysis}

To study the expression of tumor stem cell markers (CD59, CD49a, CD49d and CD133) in GSCs, flow cytometry analysis of isolated cells was performed. Cells were collected from culture dishes and after centrifugation cells were resuspended in PBS. For each sample $3 \times 10^{5}$ cells were incubated with fluorescein isothiocyanate (FITC)-conjugated antibodies for $30 \mathrm{~min}$ at room temperature and the flow-cytometry analysis was performed using fluorescence-activated cell sorting (FACS) Calibur (BD Biosciences, Franklin Lakes, NJ, USA). The results were evaluated using the BD CellQuestTM software program.

\section{Immunofluorescence staining}

In recent studies, it has been shown that CD133 is among one of the well-characterized GSC markers [12-15]. Also, nes- 

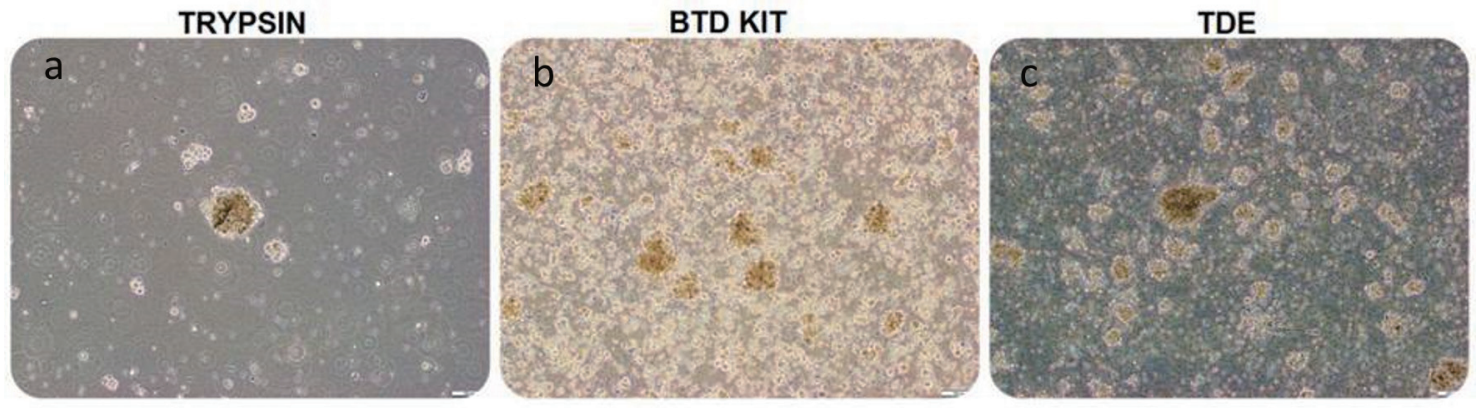

Figure 1. Glial tumor stem cells, isolated with trypsin, BTD Kit and TDE from equal amount of glioblastoma specimen, seeded $1 \times$ $10^{6}$ cells into each flask were visualized by phase-contrast microscopy on day 4, passage 0 in suspension culture (bars: a-b:100 $\mu \mathrm{m}, \mathrm{c}: 200 \mu \mathrm{m})$. Microscopic observation revealed that the number of spheres formed by trypsin-isolation (a) is lower than the other groups. The number of spheres formed by BTD Kit-isolation (b) is close to the number of spheres formed by TDE-isolated (c). BTD Kit: brain tumor dissociation Kit; TDE: tumor dissociation enzyme.

tin has been known to be as the neural stem/progenitor cell marker. Therefore, in order to determine the characterization of GSCs isolated by different methods, cells were stained with CD133 antibody (ABclonal, Manhattan Beach, CA, USA) and nestin antibody (Santa Cruz Biotech, Dallas, TX, USA) by immunofluorescence method.

The cells were seeded in eight-well chamber slides (BD Biosciences, Franklin Lakes, NJ, USA) and after $24 \mathrm{~h}$ fixed in ice-cold methanol for $10 \mathrm{~min}$. The fixated cells were washed sequentially with PBS, $0.025 \%$ Triton X-100 (Merck, Darmstadt, Germany) and PBS and then incubated with $1.5 \%$ normal goat or donkey blocking serum (Santa Cruz Biotech, Dallas, TX, USA) prepared with PBS for 20 min at room temperature. Primary antibodies (CD133 and nestin) prepared at 1:50 ratio were placed in wells and incubated for overnight at $+4{ }^{\circ} \mathrm{C}$. On the following day, samples were incubated for $2 \mathrm{~h}$ at $37^{\circ} \mathrm{C}$ and worked in the dark after this stage. Wells were washed twice with PBS and secondary antibodies (Santa Cruz Biotech, Dallas, TX, USA) were added. After waiting for $1 \mathrm{~h}$ at room temperature, the cells were washed again with PBS and dried. Samples were mounted with coverslips and mounting medium containing 4',6-diamidino-2-phenylindole (DAPI) (Santa Cruz Biotech, Dallas, TX, USA) and visualized by fluorescence microscopy.

\section{Results}

\section{Morphology of GSCs}

GCSs, which have the ability to form spheres and to adhere, have been successfully isolated from GBM tumor tissues by three different methods. Figure 1 showed that isolated cells successfully formed spheres in suspension culture by the three methods. The number of the obtained cells is different according to isolation method on passage 0 . It appears that the number of cells isolated with the first method is less than the number of cells isolated with other methods (Fig. 1a).

GCSs also proliferated by forming colony on days 4 and 12 in adherent culture and colony sizes are different according to isolation method (Fig. 2). The cells were proliferated and colonies were expanded rapidly from day 4 to day 12 . However, the proliferation of the GCSs was observed in later stages in the first method compared to the second and the third methods in which earlier proliferation of GCSs was seen (Fig. 2. a, d).

\section{Flow cytometry analysis of GSCs}

Studies have revealed that transmembrane protein CD133 expression is associated with tumor stem cells, regeneration, differentiation and is also used as a biomarker for the isolation and characterization of stem cells. Flow cytometry analysis showed that the cells isolated by all three methods have positive expression of CD133 (Fig. 3). CD59, CD49a, and CD49d used as GCS markers also had positive expressions in cells isolated by the three different methods (Fig. 3). Although the percentage of positivity of CD59 marker is high in all three methods, the percentage of CD49a in cells isolated with trypsin was low (Fig. 3, a lower panel). These cells can still be regarded as positive for CD49a since it is still above $20 \%$.

Characterization studies have shown that the isolated cells have characteristic features of GCSs.

\section{Immunofluorescence staining of glial tumor stem cells}

In addition, immunofluorescence staining was performed to characterize GCSs with CD133 and nestin. Nestin, known as a neural stem cell marker, is expressed also in glial tumor cells and its expression correlates with the malignancy potential of the glial tumor. Results of immunofluorescence staining with nestin and CD133 showed that both proteins were expressed in isolated cells by three different methods (Fig. 4). In particular, it was shown that CD133 was expressed in high amounts in cells isolated with the second method (using BTD Kit).

\section{Discussion}

The novel therapies of GBM are based on the latest cellular 

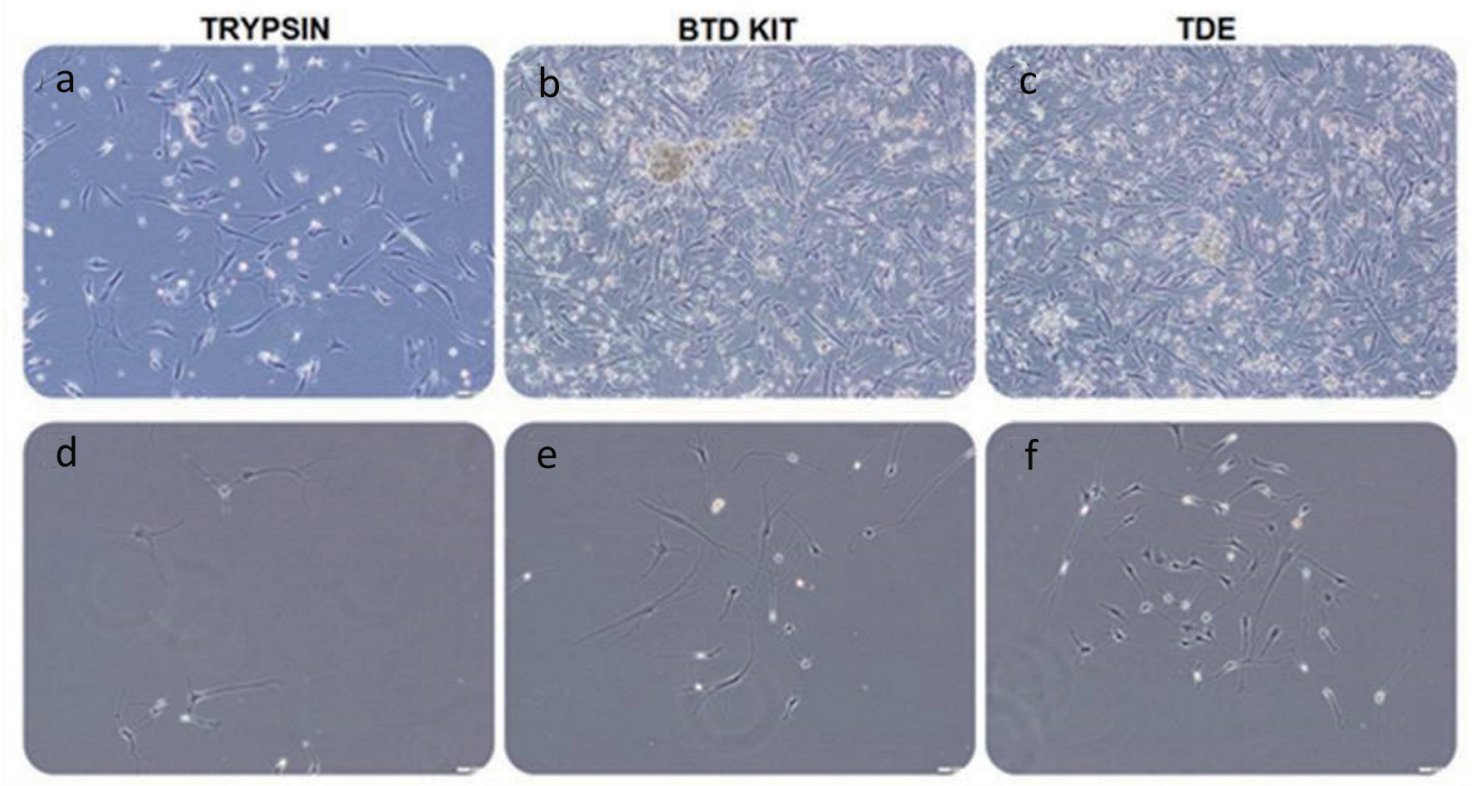

Figure 2. Glial tumor stem cells, isolated with trypsin, BTD Kit and TDE from equal amount of GBM specimen, seeded $1 \times 10^{6}$ cells into each flask were visualized by phase-contrast microscopy on day 12 (a-c) and day 4 (d-f), passage 0 in adherent culture (bars: $100 \mu \mathrm{m}$ ). Microscopic observation revealed that the number of adherent glial tumor stem cells isolated with trypsin (a, d) is less than that of other groups. The number of cells isolated with BTD Kit (b,e) is close to the number of cells isolated with TDE (c, f). BTD Kit: brain tumor dissociation Kit; TDE: tumor dissociation enzyme.

composition of the tumoral tissue. The latest cellular composition of glioblastome is characterized with proliferating blood vessels, infiltrating inflammatory cells and necrosis $[5,16]$. Both contrast enhanced magnetic resonance images and micro-morphologic differences can be observed in GBM. This multiform character originates from intra-tumoral heterogeneity $[17,18]$.

Highly heterogenous nature of GBM is the cause of therapeutic resistance $[17,19]$. These heterogenous tumoral tissues include approximately $1-3 \%$ of organizer cells in
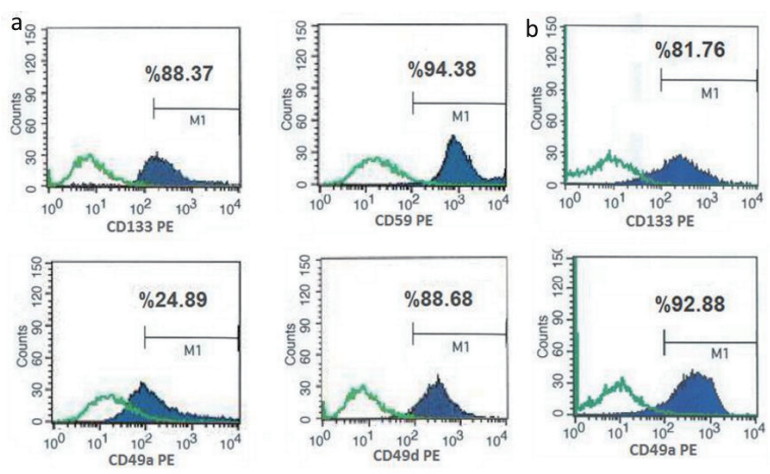

the cellular composition [16, 20]. Moreover, organizer cells show the capability of self-renewal and multi-potent differentiation. These abilities belong to stem cells and these organizer cells are called "brain tumor stem cell". Brain tumor stem cell, GSCs, GBM stem-like cells, glioma cancer stem cells and glioma initiating cells are regarded as synonyms [21].

First tumor stem cell theory was proposed by Virchow 150 years ago. According to Virchow, tumors might originate from immature cells. In the following years, Cohnheim and

Figure 3. Figure 3. Flow cytometry analyzes of glial tumor stem cells (isolated with trypsin (a), BTD Kit (b) and TDE (c)) with glial tumor stem cell markers (CD133, CD59, CD49a, CD49d). Flow cytometry analysis of collected cells from culture dishes was performed. Positive expressions of CD133, CD59, CD49a and CD49d indicate that isolated and analyzed tumor stem cells have GBM characteristics. These characteristics of isolated tumor stem cells by three different methods were compared. CD133 and CD59e expressions were found to be similar in all cells, whereas expression of CD49a was higher in cells isolated with BTD Kit, and expression of CD49d was higher in cells isolated with trypsin. This shows that these isolation methods with BTD Kit and trypsin are more successful to isolate cells which have higher expression of glial tumor stem cell markers. BTD Kit: brain tumor dissociation Kit; TDE: tumor dissociation enzyme. 

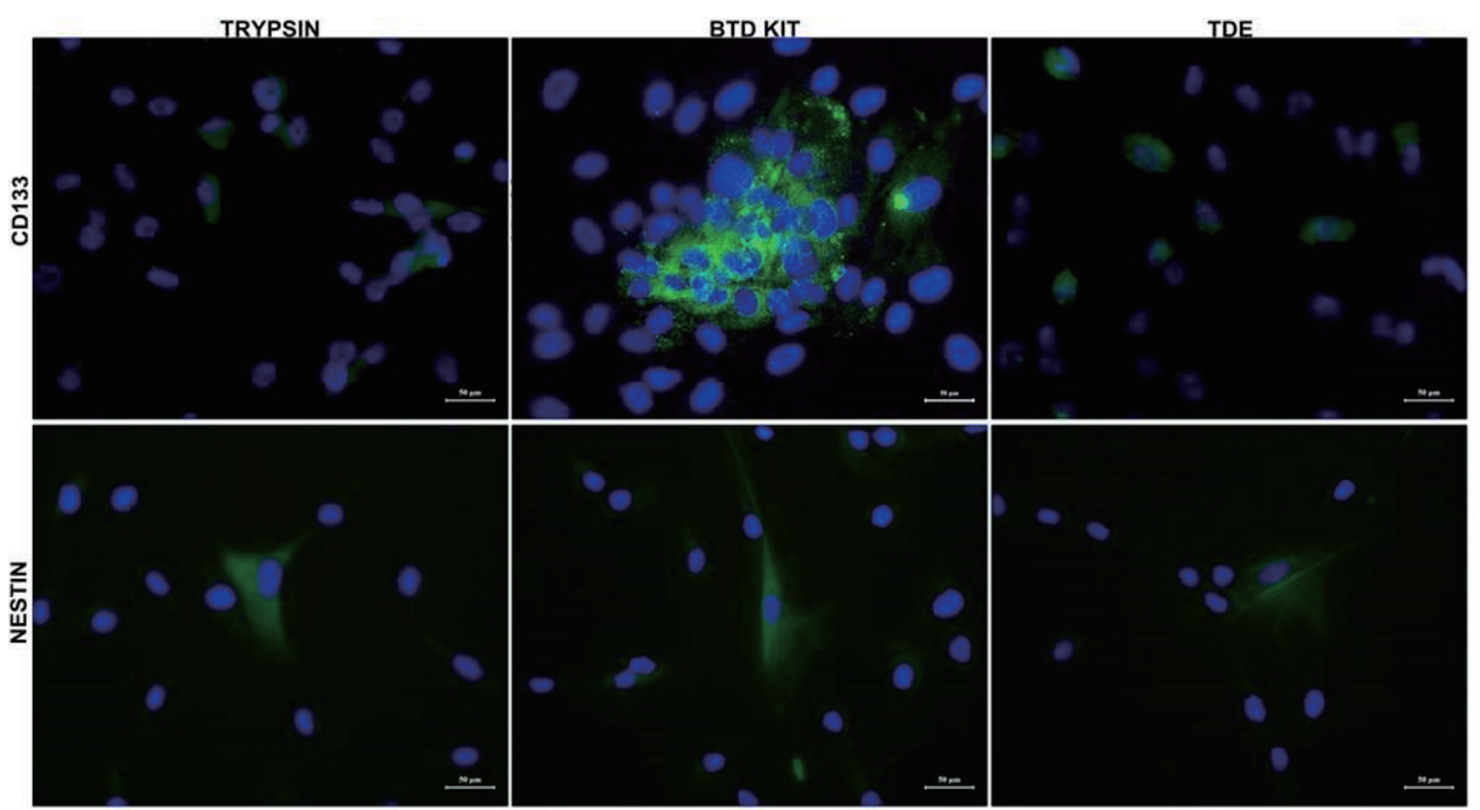

Figure 4. CD133 and nestin expression in glial tumor stem cells. Glial tumor stem cells were cultured with growth medium in chambered cell culture slides. After adhering in culture slides, cells were fixed and stained to detect CD133 and nestin. Immunofluorescence microscopy imaging of the expression of CD133 and nestin in isolated cells by three different methods are shown. Nuclei were stained with DAPI (blue) (bars: $50 \mu \mathrm{m}$ ). DAPI: 4',6-diamidino-2-phenylindole.

Durante supported the stem cell concept and suggested that adult tissues still contain dormant immature cells that could be activated and give rise to tumor development in particular conditions. Seventy years after all of these, Makino introduced the "tumor stem cell", defining them as "a small subpopulation of cells". At last, GSCs were identified directly from patientderived tumors in brain by 2000s [22, 23].

Cancer stem cells share many of the properties of normal neural stem cells. Neural stem cells are described at any stage of the development-from the embryo to the adult organism, and they are located in their specific niches. Origin of the GSCs' is not clear. Recent studies have revealed that the GSCs became after the process of "de-differentiation" from neural stem cell. These cells seem to be at the top of the hierarchy of the tumor cells and they can form GBM [16, 24].

Until now, several characteristics and markers have been identified for GSC, such as CD133 and nestin. CD133, which is a pentaspan membrane glycoprotein, has been used as a

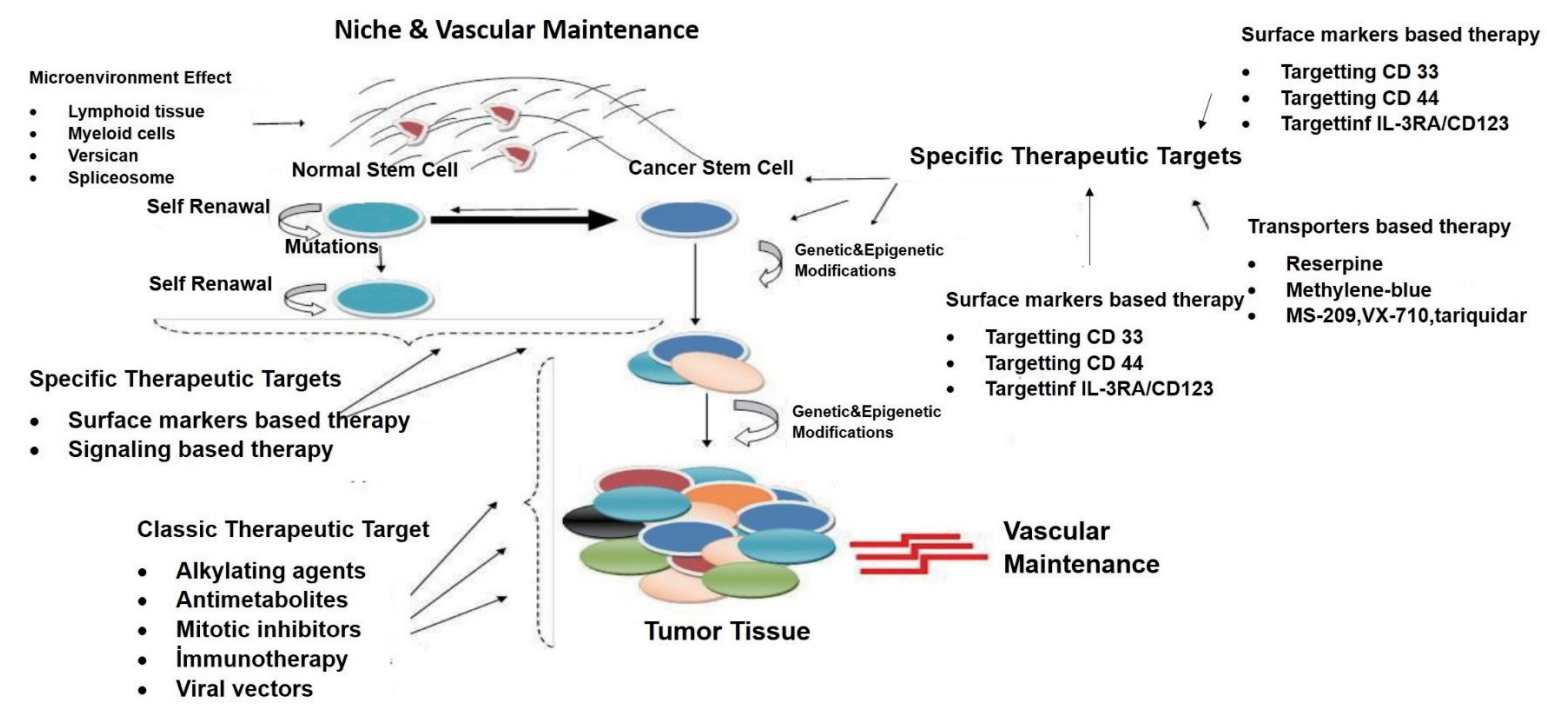

Figure 5. New therapeutic impact areas in cancer stem cell theory. This figure has showed many recent approaches to stem cell such as targeting of cell surface molecules, cell penetrating peptides, immunotherapy and change of microenvironment of niche. 
biomarker for GSCs [6]. Also, nestin, which is a cytoskeletal protein, exhibits different levels of expression in human GBM. CD15, CD44, and CD90 are also cell surface markers and are used to identify the GSCs [21].

The epidermal growth factor receptor (EGFR), aldehyde dehydrogenase 1 (ALDH1), methylguanine methyltransferase (MGMT), 1p/19q, isocitrate dehydrogenase (IDH), p53, phosphoinositide 3-kinase (PI3K), retinoblastoma protein (RB), and RAF are other well-known molecular markers in GBM. Recent studies showed that there are many of the structural proteins or transcription factors, such as oligodendrocyte transcription factor 2 (OLIG2), MYC, SOX2, MUSASHI1, NANOG, BMI1, cathepsin, embryonic leucine zipper kinase, phosphoserine phosphatase and inhibitor of differentiation protein 1 (ID1) in GSCs. Moreover, numerous aberrantly expressed genes and signaling pathways, such as EGFR, PTEN, INK4a/ARF, NF1, PDGFRA/IDH1，P53，IDH1，RB1, and ERBB2, have been identified as important measures in GBM biology. The receptor tyrosine kinase (RTK) family (EGF and PDGF), Sonic Hedgehog pathway, Notch pathway, Wnt/betacatenin pathway and their receptors are the most important key regulators of GSC's regulations, and have also been found to be altered or overexpressed in GSCs. Also, neural stem cells have been found to exhibit similar markers. There are many efforts to identify new molecular markers neural stem cells and GSCs. But the linkage between both cells is not well understood yet [6].

Nowadays, it is well-known that the poor prognosis and recurrence in GBM are mainly due to presence of GSC, which is responsible for both chemotherapy and radiotherapy. For example, several studies have shown that the percentage of $\mathrm{CD} 133^{+}$cells within malignant gliomas markedly increases following conventional chemo-radiotherapy [24, 25]. Recent analyses have focused on the individualized therapy by targeting these GSCs. Markers and signaling pathways in GSCs are possible targets in patients with GBM and these types of targeted treatments may provide more efficient therapeutic options (Fig. 5) [26].

For this purpose, many studies have been performed on specific therapies targeting both signaling pathways and activities of niches in stem cells, and activating stem cell's autophagy, immune- and viral-based therapies on stem cells [2729].

Isolation of GSCs is very critical for making research to find new mechanism of effect for GBM growth and progression and also to establish new therapeutic approaches for patients with GBM. In this study, GSC isolation was performed by three different methods and the characterization studies of the cells were carried out. Cells isolated with TDE and BTD Kit were found to grow more rapidly compared to trypsinisolated cells. Moreover, flow cytometry and immunohistochemical characterization studies were performed with CD133 marker along with other GBM markers and it was determined that cells isolated with BTD Kit was more effective in order to show the characteristic of GSCs. Based on these results, we can suggest that isolation with BTD Kit is the most effective method to isolate GSCs from GBM specimens. Further evaluation including more GBM specimens may be required in order to confirm our results.

\section{Conclusions}

Our results suggest that BID Kit method was the most appropriate method for isolating GSCs from GBM. This isolation method can be used effectively for conducting further studies to understand the molecular behavior of GBM.

\section{Acknowledgments}

None.

\section{Financial Disclosure}

This study was provided financial support in the form of SIPSC-2013-271 (Scientific Investigation Projects Support Committee) funding by Bezmialem Foundation University. It was funded by BAP grant No: B.30.2.BAV.0.05/183. The sponsor had no role in the design or conduct of this research.

\section{Conflict of Interest}

All authors certify that they have no affiliations with or involvement in any organization or entity with any financial interest (such as honoraria; educational grants; participation in speakers' bureaus; membership, employment, consultancies, stock ownership, or other equity interest; and expert testimony or patent-licensing arrangements), or non-financial interest (such as personal or professional relationships, affiliations, knowledge or beliefs) in the subject matter or materials discussed in this manuscript.

\section{Informed Consent}

Informed consent was obtained from all individual participants included in the study. Additional informed consent was obtained from all individual participants for whom identifying information is included in this article.

\section{Author Contributions}

TTD, EK, ST and MAH designed the study, collected and analyzed data, and wrote the first draft of the manuscript; ZE and ES collected data and participated in writing and revision of the manuscript; MYK and ST extracted data and revised manuscript; TTD, EK and MHS participated in the analysis of the data and revised the manuscript. All authors read and approved the final manuscript.

\section{References}

1. Glaser T, Han I, Wu L, Zeng X. Targeted nanotechnology 
in glioblastoma multiforme. Front Pharmacol. 2017;8:166.

2. Louis DN, Perry A, Reifenberger G, von Deimling A, Figarella-Branger D, Cavenee WK, Ohgaki H, et al. The 2016 World Health Organization Classification of Tumors of the Central Nervous System: a summary. Acta Neuropathol. 2016;131(6):803-820.

3. Miranda A, Blanco-Prieto MJ, Sousa J, Pais A, Vitorino C. Breaching barriers in glioblastoma. Part II: Targeted drug delivery and lipid nanoparticles. Int J Pharm. 2017;531(1):389-410.

4. Abou-Antoun TJ, Hale JS, Lathia JD, Dombrowski SM. Brain Cancer Stem Cells in Adults and Children: Cell Biology and Therapeutic Implications. Neurotherapeutics. 2017;14(2):372-384.

5. Grahovac G, Tomac D, Lambasa S, Zoric A, Habek M. Cerebellar glioblastomas: pathophysiology, clinical presentation and management. Acta Neurochir (Wien). 2009;151(6):653-657.

6. Mehta S, Lo Cascio C. Developmentally regulated signaling pathways in glioma invasion. Cell Mol Life Sci. 2018;75(3):385-402.

7. Hatiboglu MA, Wei J, Wu AS, Heimberger AB. Immune therapeutic targeting of glioma cancer stem cells. Target Oncol. 2010;5(3):217-227.

8. Yan H, Romero-Lopez M, Benitez LI, Di K, Frieboes HB, Hughes CCW, Bota DA, et al. 3D mathematical modeling of glioblastoma suggests that transdifferentiated vascular endothelial cells mediate resistance to current standardof-care therapy. Cancer Res. 2017;77(15):4171-4184.

9. Bien-Moller S, Balz E, Herzog S, Plantera L, Vogelgesang S, Weitmann K, Seifert C, et al. Association of Glioblastoma Multiforme Stem Cell Characteristics, Differentiation, and Microglia Marker Genes with Patient Survival. Stem Cells Int. 2018;2018:9628289.

10. Zhou D, Alver BM, Li S, Hlady RA, Thompson JJ, Schroeder MA, Lee JH, et al. Distinctive epigenomes characterize glioma stem cells and their response to differentiation cues. Genome Biol. 2018;19(1):43.

11. Janiszewska M, Suva ML, Riggi N, Houtkooper RH, Auwerx J, Clement-Schatlo V, Radovanovic I, et al. Imp2 controls oxidative phosphorylation and is crucial for preserving glioblastoma cancer stem cells. Genes Dev. 2012;26(17):1926-1944.

12. Brescia P, Ortensi B, Fornasari L, Levi D, Broggi G, Pelicci G. CD133 is essential for glioblastoma stem cell maintenance. Stem Cells. 2013;31(5):857-869.

13. Zhang M, Song T, Yang L, Chen R, Wu L, Yang Z, Fang J. Nestin and CD133: valuable stem cell-specific markers for determining clinical outcome of glioma patients. J Exp Clin Cancer Res. 2008;27:85.

14. Zeppernick F, Ahmadi R, Campos B, Dictus C, Helmke BM, Becker N, Lichter P, et al. Stem cell marker CD133 affects clinical outcome in glioma patients. Clin Cancer Res. 2008;14(1):123-129.
15. Wu Y, Wu PY. CD133 as a marker for cancer stem cells: progresses and concerns. Stem Cells Dev. 2009;18(8):1127-1134.

16. Kyurkchiev DS. Cancer stem cells from glioblastoma multiforme: culturing and phenotype. OA Stem Cells. 2104;2:3-9.

17. Brooks LJ, Parrinello S. Vascular regulation of glioma stem-like cells: a balancing act. Curr Opin Neurobiol. 2017;47:8-15.

18. Wang J, Ma Y, Cooper MK. Cancer stem cells in glioma: challenges and opportunities. Transl Cancer Res. 2013;2(5):429-441.

19. Altaner C. Glioma cancer stem cells and their role in therapy. Atlas Genet Cytogenet Oncol Haematol. 21012;16:757-764.

20. Pavon LF, Sibov TT, de Oliveira DM, Marti LC, Cabral FR, de Souza JG, Boufleur P, et al. Mesenchymal stem cell-like properties of CD133+ glioblastoma initiating cells. Oncotarget. 2016;7(26):40546-40557.

21. Ludwig K, Kornblum HI. Molecular markers in glioma. J Neurooncol. 2017;134(3):505-512.

22. D'Andrea V, Guarino S, Di Matteo FM, Maugeri Sacca M, De Maria R. Cancer stem cells in surgery. G Chir. 2014;35(11-12):257-259.

23. Ignatova TN, Kukekov VG, Laywell ED, Suslov ON, Vrionis FD, Steindler DA. Human cortical glial tumors contain neural stem-like cells expressing astroglial and neuronal markers in vitro. Glia. 2002;39(3):193-206.

24. Safa AR, Saadatzadeh MR, Cohen-Gadol AA, Pollok KE, Bijangi-Vishehsaraei K. Glioblastoma stem cells (GSCs) epigenetic plasticity and interconversion between differentiated non-GSCs and GSCs. Genes Dis. 2015;2(2):152163.

25. Tamura K, Aoyagi M, Wakimoto H, Ando N, Nariai T, Yamamoto M, Ohno K. Accumulation of CD133-positive glioma cells after high-dose irradiation by Gamma Knife surgery plus external beam radiation. J Neurosurg. 2010;113(2):310-318.

26. Mitra AK, Agrahari V, Mandal A, Cholkar K, Natarajan C, Shah S, Joseph M, et al. Novel delivery approaches for cancer therapeutics. J Control Release. 2015;219:248268.

27. Fan X, Khaki L, Zhu TS, Soules ME, Talsma CE, Gul N, Koh C, et al. NOTCH pathway blockade depletes CD133positive glioblastoma cells and inhibits growth of tumor neurospheres and xenografts. Stem Cells. 2010;28(1):516.

28. Kim SS, Rait A, Kim E, Pirollo KF, Nishida M, Farkas N, Dagata JA, et al. A nanoparticle carrying the p53 gene targets tumors including cancer stem cells, sensitizes glioblastoma to chemotherapy and improves survival. ACS Nano. 2014;8(6):5494-5514.

29. Matsui WH. Cancer stem cell signaling pathways. Medicine (Baltimore). 2016;95(1 Suppl 1):S8-S19. 